(Research Article)

\title{
Sever Oligospermia Treatment with Testicular Sperm Using ICSI
}

\begin{abstract}
Mustafa Zakaria*1, Aya Al-ibraheemi ${ }^{2 *}$, Mohammed Ennaji ${ }^{3}$, Wassym R. Senhaji ${ }^{4}$, Mohammed Zarqaoui ${ }^{5}$, Hayder A. Mossa ${ }^{6}$, Modou Mamoune Mbaye ${ }^{7}$ and Noureddine Louanjli ${ }^{8}$.
\end{abstract}

${ }^{1}$ MD. Senior Clinical Embryology and Assisted Reproductive, Deputy Executive Director and Administrative Coordinator of the Association for Scientific Research of the IRIFIV-AISRG Group, Consultant in IRIFIV Fertility Center, Casablanca, Morocco

${ }^{2}$ Embryologist, UK. Researcher in the Scientific Research Group - and Member of the Association for Scientific Research of the IRIFIV-AISRG Group - UK

${ }^{3}$ Embryologist, Member in of the Association for Scientific Research of the IRIFIV-AISRG Group Casablanca, Morocco

${ }^{4} G Y N$ OBT, Member in of the Association for Scientific Research of the IRIFIV-AISRG Group Casablanca, Morocco

${ }^{5}$ GYN OBT, Head of the Association for Scientific Research of the IRIFIV-AISRG Group Casablanca, Morocco

${ }^{6}$ High Institute for Infertility Diagnosis and Assisted Reproductive Technologies Al-Nahrain University, Baghdad, IRAQ" Scientific Research in Clinical Embryology, Laboratory ART's and Reproductive Physiology and Member of the Association for Scientific Research of the IRIFIVAISRG Group - IRAQ

${ }^{7}$ Laboratory of Physiopathology, Genetics Molecular and Biotechnology (PGMB), Faculty of Sciences Ain Chock, Research Center. Health and Biotechnology, University Hassan II - Research Associate at the fertility center Irifiv a rsearcher in the Scientifi Research Group-Casablanca, Morocco

${ }^{8}$ Head of the LABOMAC Laboratory, and IRIFIV Fertility Center, AFC Fertility Center, Casablanca, Morocco 


\section{Corresponding author :}

Mustafa Zakaria: MD. Senior Clinical Embryology and Assisted Reproductive, Deputy Executive Director and Administrative Coordinator of the Association for Scientific Research of the IRIFIVAISRG Group, Consultant in IRIFIV Fertility Center, https://orcid.org/0000-0002-3087-5604 Casablanca, Morocco.

info@irifiv-aisrg.com /dr.zakaria@irifiv-aisrg.com

Tel; +212522397339|+212669856255 Fax: +212522268303

\section{Abstract}

Assisted reproductive technology has been developed significantly throughout the past few years, particularly diagnosing and treating male infertility. Many studies have been performed showing that Intracytoplasmic Sperm Injection (ICSI) is a successful method to attain clinical pregnancy and live birth through impaired spermatozoa characteristics or low sperm count, such as severe oligospermia. Severe oligospermia indicates low sperm count, which in some cases leads to azoospermia. Severe oligospermia can be caused by several factors such as genetics or medication. In search of efficient treatment for couples with Severe oligospermia, numerous retrospective and prospective researches have reported high pregnancy and live birth rates through testicular sperm for men with severe oligospermia and cryptozoospermia with or without high sperm DNA damage. The research showed that the use of testicular sperm in combination with ICSI yielded a high pregnancy rate and live births over another source of sperm, such as ejaculated sperms. Moreover, the use of ICSI in severe oligospermia has shown successful fertilization and pregnancy.

\section{Abbreviations}

Gonadotropin-releasing hormone (GnRH) ,Intracytoplasmic Sperm Injection (ICSI) ,gonadotropin-releasing hormone $(\mathrm{GnRH})$, luteinizing hormone (LH), follicle-stimulating hormone (FSH), Y chromosome (Yq), azoospermia factor (AZF), Cystic fibrosis transmembrane conductance regulator (CFTR), oligo-astheni-teratozoospermia (OAT), assisted reproductive technology (ART) ,DNA fragmentation index (DFI) ,sperm DNA fragmentation (SDF),

\section{Keywords}


Sever oligospermia, Medication and cancer treatment influence on oligozoospermia, ICSI for men with sever oligozoospermia.

\section{Introduction}

Male infertility factors have been recognised in around $40 \%-50 \%$ of infertile couples; more than $90 \%$ of cases in male infertility are due to low sperm counts, poor sperm quality or both. Other factors involve anatomical disorders, hormonal imbalances and genetic defects [1] (Leaver, 2016). Moreover, male factors can include ejaculatory problems such as retrograde ejaculation, premature ejaculation or anejaculation, defects in sperm count, mobility, or abnormalities [1] (Leaver, 2016). Some of the most common factors that correlate to irregularities in sperm are listed in (Table 1).

Male infertility has geographical variation in the prevalence of male infertility. High fertility levels can be found in France (59\%), $26 \%-32 \%$ in the UK and Kashmir Valley in India, and about 36 $\%$ in South Africa, Indonesia and Finland. Moreover, it has been shown that mean sperm concentration has regional variation in men from different regions of the USA and France [2] (Mehta et al., 2006).

Sperm Abnormalities

Aspermia

Azoospermia

Oligozoospermia

Oligospermia

Teratozoospermia

\section{Descriptions}

Male with no semen

Male with no sperms in the semen

Low sperm concentration

Low sperm count

Irregular sperm shape ( $96 \%$ of sperms)

Table 1: Sperm abnormalities, and factors that lead to male infertility (Leaver, 2016).

There are different ways of male infertility treatment, depending on the male infertility factors, for instance, improving the quality of sperm, surgery to repair a varicocele or intracytoplasmic sperm injection (ICSI) procedures. ICSI requires injecting one sperm directly into an oocyte prior to transferring to the uterus. ICSI has the advantage of using few viable sperm to fertilise an egg and can bypass natural barriers that prevent fertilisation [1] (Leaver, 2016).

This report will be discussing and evaluating the application of ICSI in treating men with significant oligospermia 


\section{Sever oligospermia}

Oligozoospermia is a disorder where sperm concentration is below the lower reference limit of 15 million sperm $/ \mathrm{ml}$ of ejaculate. Oligozoospermia can be further classified as mild (between 10 and 15 million sperm $/ \mathrm{ml}$ ), moderate oligozoospermia (between 5 and 10 million sperm $/ \mathrm{ml}$ ), and severe oligozoospermia (less than 5 million sperm/ml) [3] (Choy and Amory, 2020). Oligozoospermia diagnosing can be challenging, as several conditions are recognised to result in oligozoospermia. Therefore, a formal and precise diagnosis can be relevant in giving patients a reason for their sterility and decreasing confusion, fear, and stigma [3] (Choy and Amory, 2020).

Disturbance of the regulated hormonal axis can induce the increase to several of the endocrinebased etiologist of oligozoospermia. Usually, the hypothalamus produces and secretes gonadotropin-releasing hormone $(\mathrm{GnRH})$ into the hypothalamic-hypophyseal portal circulation; GnRH influences the anterior pituitary to induce the gonadotroph cells to produce and release luteinizing hormone $(\mathrm{LH})$ and follicle-stimulating hormone (FSH) into the systemic circulation. LH excites the Leydig cells of the testes to deliver testosterone, while FSH stimulates the Sertoli cells to induce spermatogenesis (Figure 1). Generally, any destruction of gonadotropin secretion will result in a downstream outcome of disrupting intratesticular testosterone biosynthesis and spermatogenesis [3] (Choy and Amory, 2020).

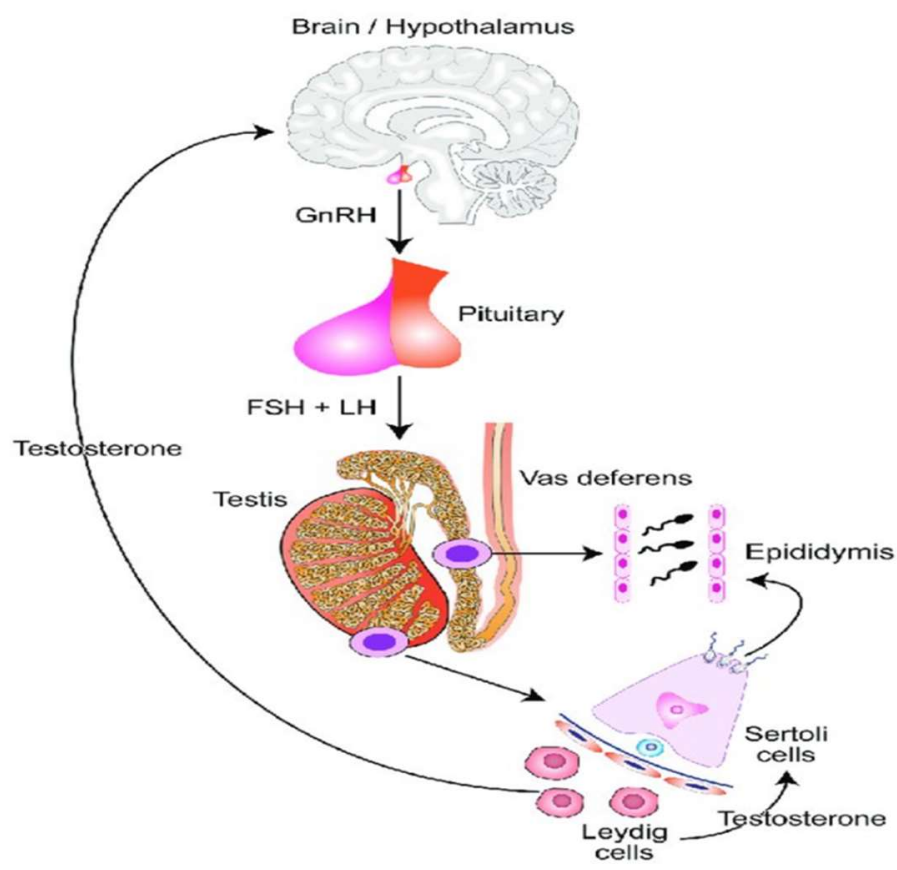

Figure 1: The hypothalamic-pituitary-testicular axis (Reynolds-Wright and Anderson, 2019). 
Males with infertility have higher chromosomal irregularities than fertile men and usually do not present any phenotypic features. In the oligozoospermic, men of autosomal translocations have a rate of inversions predominate $(3 \%)$ over sex chromosome anomalies (1.6\%). Fertility may be expected, but interference with spermatocyte chromosomal pairing re- results in azoospermia or, more frequently, oligozoospermia (McLachlan, 2013)

Yq microdeletions are the most well-known genetic condition of spermatogenic failure. Microdeletions of the Y chromosome (Yq) include the azoospermia factor (AZF) regions with several copies of spermatogenic genes. The high oligozoospermic rate of approximately $4 \%$ of men with sperm densities of $0.1-5$ million $/ \mathrm{ml}$. The AZFc deletion accounts for about $60 \%$ of $Y$ microdeletions, and of these men, one-third have severe oligozoospermia. Moreover, the high homology within palindromic sequences induces intra- chromosomal recombination and deletions of 0.8-7.7 megabase segments that arise in embryonic life [4] (McLachlan, 2013).

AZF is divided into regions $\mathrm{a}, \mathrm{b}$, and $\mathrm{c}$ in the $\mathrm{Y}$ chromosome. Complete AZF deletions rates in the general population are rare $(1$ in 4,000); however, they happen in $10 \%$ of patients with idiopathic nonobstructive azoospermia and 5\% of men with severe oligozoospermia (mainly with a male who has $<2$ million spermatozoa per ml). Another factor of AZF mutation is the carriage of NAHR substrates inside the AZFc region, which can arise the development of various partial AZFc deletions. The most clinically relevant NAHR deletion is the gr/gr deletion for its frequency. NAHR deleted half of the AZFc region gene content, which resulted in a substantially increased risk of a male having oligozoospermia [5] (Krausz and Riera-Escamilla, 2018).

Moreover, constitutional chromosome irregularities are also a common cause of male infertility, detected in up to $20 \%$ of infertile men with oligozoospermia. The aberrations involve numerical defects, like XYY karyotype in Klinefelter syndrome, its alternatives and structural rearrangements, Robertsonian translocations, balanced reciprocal translocations, and inversions [4] (Zorrilla and Yatsenko, 2013). 47, XYY syndrome can also be a factor of oligozoospermia; it has been recognised that germ cells with an extra Y chromosome from a male with the 47 XYY karyotypes have odd meiotic pairing, indicating disturbed meiosis, oligozoospermia and infertility. Furthermore, structural chromosomal irregularities are found in patients with azoospermia and oligozoospermia, as autosomal defects are more common in oligozoospermia. (SCAs) include deletions, duplications, translocations, and inversions [4] (Zorrilla and Yatsenko, 2013).

Cystic fibrosis transmembrane conductance regulator (CFTR) gene mutations can induce oligozoospermia, associated with inherited infertility. Mutations in the (CFTR) gene frequently lead to male infertility. Most frequently, CFTR mutations point to a congenital bilateral loss of the vas deferens and severe oligozoospermia. Mutations in the CFTR gene is associated with obstruction of the male genital tract rather than primary testicular failure (Dohle, 2002).

\section{Medication and cancer treatment influence on oligozoospermia}

Medications are identified to affect the hypothalamic-pituitary-testicular axis functioning and could lead to infertility from oligozoospermia. For example, endocrine influences of long-term opioid therapy include suppression of the hypothalamic-pituitary-gonadal axis via suspension of hypothalamic pulsatile GnRH secretion, which results in hypogonadism, sexual dysfunction, and infertility [6] (de Vries et al., 2019). Opioid high doses and long duration of opioid therapy are 
correlated with an increased rate of opioid-induced hypogonadism, though the impact points to be reversible following opioid discontinuation; as a result, this distribution can lead to oligozoospermia [6] (de Vries et al., 2019).

Cancer treatments effectiveness increase if a mixture of molecules is used, such as mechlorethamine, vincristine, procarbazine, and prednisone) and ABVD (adriamycin, bleomycin, vinblastine, and dacarbazine) to treat cancer such as Hodgkin's lymphoma [7] (Delessard et al., 2020). Moreover, the medication regimen taken by children, adolescents, and young adults include alkylating agents (e.g., chlorambucil, cyclophosphamide, cisplatin, busulphan). Most chemotherapy molecules are associated with a high risk of infertility, that is, according to the degree of spermatogenesis damage ranging from oligozoospermia to non-obstructive azoospermia after recovery [7] (Delessard et al., 2020).

\section{ICSI for men with sever oligozoospermia}

New researches have investigated the potential of ICSI to overcome oligozoospermia; in cases of male subfertility, ICSI treatment results in high fertilization rates per oocyte compared with conventional IVF treatment. Also, it has been shown that severe patients of oligo-astheniteratozoospermia (OAT) can now be successfully treated with ICSI [8] (Hashimoto et al., 2010).

Furthermore, another research study the correlation between prewash progressive sperm motility and pregnancy rate in severely oligoasthenozoospermic patients in (ICSI) cycle. The patients were classified into two groups depending on prewash progressive sperm motility [9] (TURHAN, PEKEL, AYRIM and BAYRAK, 2011). Group one had progressive sperm motility under 10\%, and group two had progressive sperm motility equal to or greater than $10 \%$. The result shows no significant differences among the two groups regarding the total number of oocytes retrieved, number of mature oocytes, fertilization rate, or the number of transferred embryos. In group two, the clinical pregnancy rate was significantly higher where sperm motility was higher $(62.5 \%$ [25/40] than in group I (32.5\% [13/40]), with $\mathrm{P}=0.014$ (TURHAN, PEKEL, AYRIM and BAYRAK, 2011) [9].

Mukhtar et al., 2017 evaluate assisted reproductive technology (ART) in treating male infertility; patients were treated using different types of assisted reproductive techniques such as IUI, IVF or ICSI. IVF had the highest pregnancy rate among other ART treatments (46.4\%) compared to ICSI and IUI, 35.5 and 16\%, respectively. Moreover, in a patient with oligospermia, the pregnancy outcome among male partners was $47.8 \%, 36.4 \%$ and $20 \%$ in IVF, ICSI, and IUI, respectively (Mukhtar, Shaman, Mirghani and Almasalmah, 2017) [10] (Figure 2). 


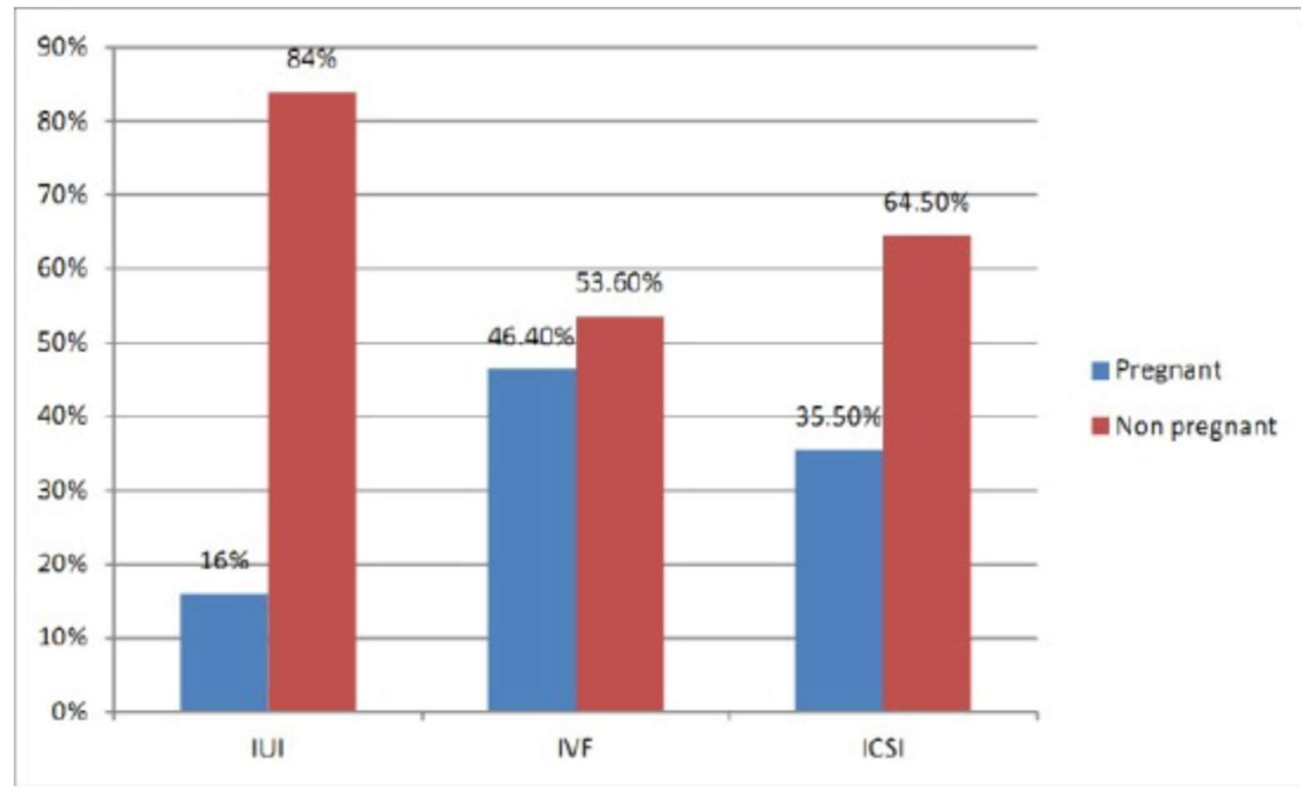

Figure 2: ART outcomes to treat male infertility, where IVF had the highest pregnancy rate (Mukhtar, Shaman, Mirghani and Almasalmah, 2017).

Sperm parameter, such as morphology and motility impact fertilization and pregnancy rate, in addition to sperm DNA integrity, which is considered to be a major factor of the quality of the injected sperm. Male infertility with high sperm DNA fragmentation index (DFI) has result in poor ICSI outcomes with lower fertilization rate, pregnancy rate, live birth rate, and higher abortion rate (HM, 2019) [11]. HM, 2019, conducted a study to compare (ICSI) results in patients with severe oligospermia using testicular vs ejaculated spermatozoa. As testicular sperm has been shown to have considerably less DNA fragmentation than other sperm sources; as a result, the use of the testicular sperm with its higher DNA integrity can generate higher success rates in oligospermia men (HM, 2019). The clinical pregnancy percentage was significantly higher in group B, where testicular spermatozoa were injected $(49.50 \%$ vs $35.57 \%, \mathrm{p}=0.044 *)$. Still, there was no significant difference in the fertilization rate between group A and B (group A 67.93\%, group B 68.01\%, $\mathrm{p}=0.960$ ). Moreover, high-quality embryos were found to be higher in the testicular sperm group (HM, 2019) [11].

Testicular sperms strength relies on robust chromatin integrity since the main pathways leading to sperm DNA fragmentation are induced through sperm transport into the seminiferous tubules or epididymis transit (Esteves, Roque and Garrido, 2018) [12]. The validity of this biological aspect relies on, firstly, chromatin compaction is continuous throughout epididymal transit. Secondly, high reactive oxygen species can be produced in the epithelial cells of epididymis under physicochemical stressors like high temperature and environmental circumstances. Finally, the DNA of mature live sperm can be cleaved by endonucleases. Consequently, sperm DNA fragmentation can occur within various pathways, revealing the high rate of SDF in live ejaculated sperm (Figure 3) (Esteves, Roque and Garrido, 2018) [12]. 


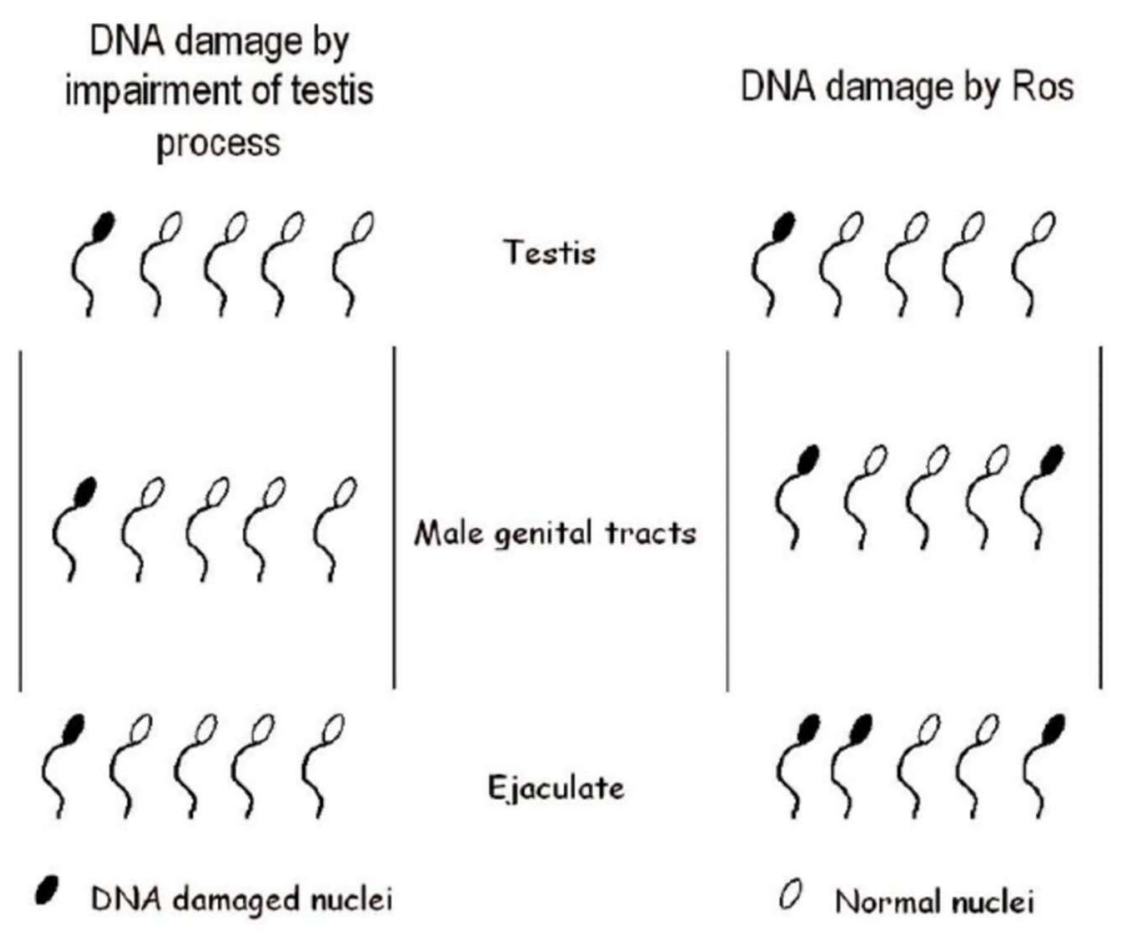

Figure 3: Origin of sperm and DNA fragmentation rate, as sperm DNA damage, can arise only in the testis and increase post testicular site (Muratori, 2006).

Therefore, testicular sperm collected through testicular sperm aspiration (TESA) or extraction (TESE) have the potential of using and selecting spermatozoa free of DNA damage for ICSI (Figure 4).

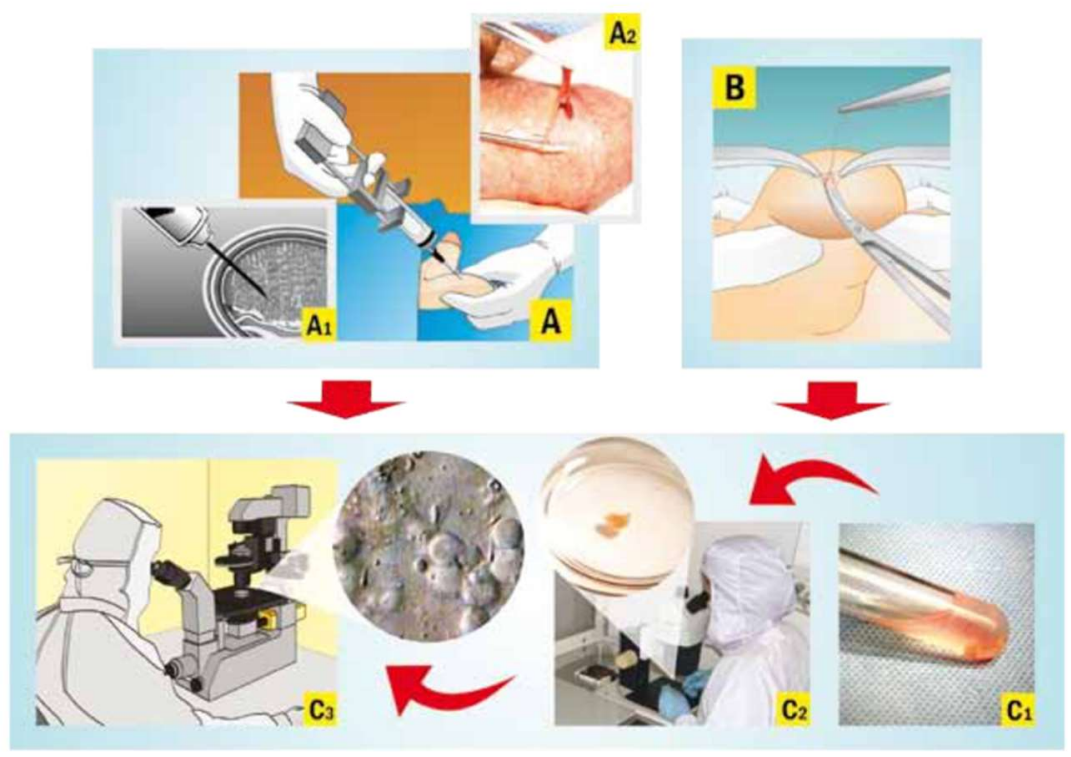


Figure 4: Overview of TESA procedure. A) Percutaneous Testicular Sperm Aspiration where a small biopsy is aspirated of the testis tissue using a needle attached to a syringe ( $A 1, A 2)$. In $B$, conventional Testicular Sperm Extraction (TESE) is performed to open scrotal layers down to the albuginea. In C1, Testicular biopsy is flushed into the sperm media tube, then the testicular biopsy is washed free from blood clots, and seminiferous tubules are mechanically dispersed (C2); finally, the testicular homogenates are examined under the microscope to prove the presence of spermatozoa (Esteves, Miyaoka and Agarwal, 2011) [13].

Besides, oocyte fertilization by a gnomically intact testicular spermatozoon will increase the possibilities of forming a normal embryonic genome that will eventually improve the likelihood of pregnancy and live birth (Esteves, Roque and Garrido, 2018) [14].

Furthermore, as mentioned before, differences observed in fertility outcomes in male infertility treatment are due to the sperm DNA fragmentation level between ejaculated and testicular sperm. For instance, the results of terminal deoxynucleotidyl transferase-mediated dUTP nick-end labelling (TUNEL) positive sperm with DNA fragmentation is lower in the testicular sample compared with ejaculated samples. The use of testicular sperm for ICSI was associated with had 50\% pregnancy and live birth rate (Mehta, Bolyakov, Schlegel and Paduch, 2015) [15].

Esteves et al., 2015, evaluate the potential of ICSI using testicular sperm to treat infertility in men with high sperm DNA fragmentation (SDF) and oligozoospermia. Sperm DNA fragmentation rate was almost 5-fold less in testicular sperm than in ejaculated sperm, where the usage of testicular sperm was associated with improved ICSI results in men with oligozoospermia and high SDF (Esteves et al., 2015) [15].

Esteves et al., 2015, evaluate the potential of ICSI using testicular sperm to treat infertility in men with high sperm DNA fragmentation (SDF) and oligozoospermia. Sperm DNA fragmentation rate was almost 5-fold less in testicular sperm than in ejaculated sperm, where the usage of testicular sperm was associated with improved ICSI results in men with oligozoospermia and high SDF (Esteves et al., 2015) [15]. Testicular sperm retrieval produced motile sperm for injections in all cases; accordingly, the sperm DNA fragmentation index was $8.3 \%$ in testicular sperm, compared to $40.7 \%$ in ejaculated sperm. For the testicular sperm - ICSI group, the clinical pregnancy rate was $51.9 \%$ versus $40.2 \%$ in the ejaculated sperm - ICSI group, the miscarriage rate was $10.0 \%$ for testicular sperm retrieval 34.3\% in ejaculated sperm (Esteves et al., 2015).

In the Greco et al., 2005 study, the DNA fragmentation rate in the testicular sperm samples was $3.6 \%$, significantly lower than the ejaculated sperm samples from the same individuals $(23.6 \%)$. Moreover, the use of testicular spermatozoa for ICSI decreases the reproductive disadvantage associated with the use of ejaculated spermatozoa for ICSI in oligozoospermia patients (Table 2) (Greco et al., 2005) [16.17.18.19] 


\begin{tabular}{lllllll}
$\begin{array}{l}\text { Sperm } \\
\text { source }\end{array}$ & Attempts & $\begin{array}{l}\text { Oocytes } \\
\text { injected }\end{array}$ & $\begin{array}{l}\text { Normal } \\
\text { zygotes }^{\mathrm{a}}\end{array}$ & $\begin{array}{l}\text { Fertilization } \\
\text { rate }^{\mathrm{b}}\end{array}$ & $\begin{array}{l}\text { Cleaved } \\
\text { embryos }^{\mathrm{c}}\end{array}$ & $\begin{array}{l}\text { Good-morphology } \\
\text { embryos }^{\mathrm{d}}\end{array}$ \\
\hline $\begin{array}{l}\text { Ejaculate } \\
\text { Testis }\end{array}$ & 18 & 185 & 131 & $70.8 \%$ & $124(94.7 \%)^{\mathrm{e}}$ & $59(47.6 \%)^{\mathrm{e}}$ \\
\hline
\end{tabular}

Table 2: Fertilization and embryo development after ICSI with ejaculated and testicular spermatozoa (Greco et al., 2005) [16.17.18.19]

\section{Conclusion :}

Sever oligospermia indicate low sperm count, which can lead to male infertility; severe oligospermia be can overcome through ICSI. Genetic factors like microdeletions of the Y chromosome (Yq) can cause severe oligospermia or chemotherapy molecules, affecting the sperm count directly. The research results indicate that ICSI effectively treats severe oligospermia, mainly if testicular sperms are used through TESA. Since testicular sperms have a robust chromatin integrity, which can save sperms from DNA fragmentation in cases of sever oligospermia.

\section{Acknowledgements}

Objective of the Association for Scientific Research of the IRIFIV-AISRG Reproductive Medicine and Endometriosis Research Group (IRIFIV-AISRG), Research foundation in Casablanca, Maintaining consistent and reliably high success rates is a monthly challenge for in IVF labs, the IRIFIV Fertility Center in Casablanca - Morocco Department of Reproductive Medicine and Reproductive Biology and Embryology, advocacy of interdisciplinary Department of Reproductive Medicine and Reproductive Biology and Embryology study, encompassing the areas of research, collections and publishing Articles.

\section{Conflicts of Interest}

The authors declare no conflicts of interest regarding the publication of this paper 


\section{References}

1. Choy, J. and Amory, J., 2020. Nonsurgical Management of Oligozoospermia. The Journal of Clinical Endocrinology \& Metabolism, 105(12), pp.e4194-e4207.

2. de Vries, F., Bruin, M., Lobatto, D., Dekkers, O., Schoones, J., van Furth, W., Pereira, A., Karavitaki, N., Biermasz, N. and Zamanipoor Najafabadi, A., 2019. Opioids and Their Endocrine Effects: A Systematic Review and Meta-analysis. The Journal of Clinical Endocrinology \& Metabolism, 105(4), pp.1020-1029.

3. Delessard, M., Saulnier, J., Rives, A., Dumont, L., Rondanino, C. and Rives, N., 2020. Exposure to Chemotherapy During Childhood or Adulthood and Consequences on Spermatogenesis and Male Fertility. International Journal of Molecular Sciences, 21(4), p. 1454 .

4. Dohle, G., 2002. Genetic risk factors in infertile men with severe oligozoospermia and azoospermia. Human Reproduction, 17(1), pp.13-16.

5. Esteves, S., Miyaoka, R. and Agarwal, A., 2011. Sperm retrieval techniques for assisted reproduction. International braz j urol, 37(5), pp.570-583.

6. Esteves, S., Sánchez-Martín, F., Sánchez-Martín, P., Schneider, D. and Gosálvez, J., 2015. Comparison of reproductive outcome in oligozoospermic men with high sperm DNA fragmentation undergoing intracytoplasmic sperm injection with ejaculated and testicular sperm. Fertility and Sterility, 104(6), pp.1398-1405.

7. Esteves, S., Roque, M. and Garrido, N., 2018. Use of testicular sperm for intracytoplasmic sperm injection in men with high sperm DNA fragmentation: a SWOT analysis. Asian Journal of Andrology, 20(1), p.1.

8. Hashimoto, H., Ishikawa, T., Goto, S., Kokeguchi, S., Fujisawa, M. and Shiotani, M., 2010. The Effects of Severity of Oligozoospermia on Intracytoplasmic Sperm Injection (ICSI) Cycle Outcome. Systems Biology in Reproductive Medicine, 56(1), pp.91-95.

9. HM, M., 2019. Reproductive Outcomes in Patients with Severe Oligospermia Undergoing Intracytoplasmic Sperm Injection using Testicular Versus Ejaculated Spermatozoa. Investigations in Gynecology Research \& Womens Health (IGRWH), 3(1).

10. Krausz, C. and Riera-Escamilla, A., 2018. Genetics of male infertility. Nature Reviews Urology, 15(6), pp.369-384.

11. Leaver, R., 2016. Male infertility: an overview of causes and treatment options. British Journal of Nursing, 25(18), pp.S35-S40.

12. McLachlan, R., 2013. Approach to the Patient With Oligozoospermia. The Journal of Clinical Endocrinology \& Metabolism, 98(3), pp.873-880. 
13. Mehta, A., Bolyakov, A., Schlegel, P. and Paduch, D., 2015. Higher pregnancy rates using testicular sperm in men with severe oligospermia. Fertility and Sterility, 104(6), pp.13821387.

14. Mehta, R., Makwana, S., Ranga, G., Srinivasan, R. and Virk, S., 2006. Prevalences of oligozoospermia and azoospermia in male partners of infertile couples from different parts of India. Asian Journal of Andrology, 8(1), pp.89-93.

15. Mukhtar, H., Shaman, A., Mirghani, H. and Almasalmah, A., 2017. The Outcome of Assisted Reproductive Techniques among Couples with Male Factors at Prince Khalid Bin Sultan Fertility Centre, Kingdom of Saudi Arabia. Open Access Macedonian Journal of Medical Sciences, 5(5), pp.603-607.

16. Muratori, M., 2006. Origin and biological significance of DNA fragmentation in human spermatozoa. Frontiers in Bioscience, 11(1), p.1491.

17. Reynolds-Wright, J. and Anderson, R., 2019. Male contraception: where are we going and where have we been?. BMJ Sexual \& Reproductive Health, 45(4), pp.236-242.

18. TURHAN, N., PEKEL, A., AYRIM, A. and BAYRAK, Ö., 2011. ICSI outcome in severely oligoasthenozoospermic patients and its relationship to prewash progressive sperm motility. Turk J Med Sci,.

19. Zorrilla, M. and Yatsenko, A., 2013. The Genetics of Infertility: Current Status of the Field. Current Genetic Medicine Reports, 1(4), pp.247-260. 